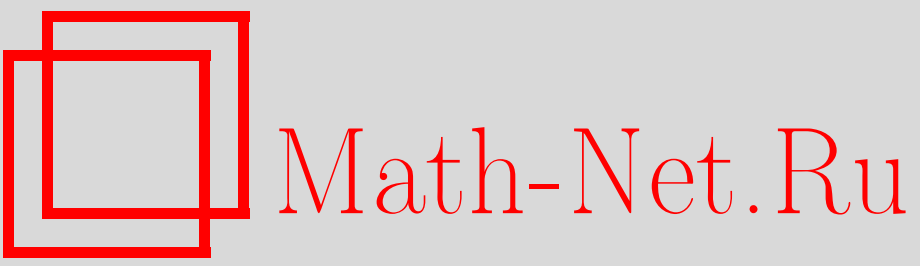

А. П. Янковский, Расчёт установившейся ползучести металлокомпозитных пологих оболочек слоисто-волокнистой структуры, Вестн. Сам. гос. техн. ун-та. Сер. Физ.-мат. науки, 2010, выпуск 1(), 71-83

DOI: https://doi.org/10.14498/vsgtu741

Использование Общероссийского математического портала Math-Net.Ru подразумевает, что вы прочитали и согласны с пользовательским соглашением

http://www.mathnet.ru/rus/agreement

Параметры загрузки:

IP : 52.6 .47 .48

26 апреля 2023 г., $07: 56: 31$ 
УДК 539.376

\title{
РАСЧЁТ УСТАНОВИВШЕЙСЯ ПОЛЗУЧЕСТИ МЕТАЛЛОКОМПОЗИТНЫХ ПОЛОГИХ ОБОЛОЧЕК СЛОИСТО-ВОЛОКНИСТОЙ СТРУКТУРЫ
}

\section{А. П. Янковский}

Институт теоретической и прикладной механики им. С. А. Христиановича СО РАН, 630090, Новосибирск, ул. Институтская, 4/1.

E-mails: nemirov@itam.nsc.ru, shulgin@itam.nsc.ru

\begin{abstract}
Сформулирована задача деформирования слоистых пологих оболочек переменной толщины, армированных волокнами постоянного поперечного сечения и работающих в условиях установившейся ползучести всех фаз композииии. Проанализирована система разрешающих уравнений и соответствующие ей граничные условия. Разработан метод решения поставленной задачи. Указан путь приближённого решения таких задач в условиях неустановившейся ползучести. Проведены конкретные расчёты, показавшие, что податливость тонкостенных конструкций в условиях установившейся ползучести существенно зависит от структуры армирования.
\end{abstract}

Ключевые слова: металлокомпозиты, армирование, оболочки пологие, пластины, слоисто-волокнистые структуры, установившаяся ползучесть.

Введение. Настоящая работа продолжает исследования, опубликованные автором в $[1,2]$, где рассматривалась проблема расчёта металлокомпозитных пластин слоисто-волокнистой структуры, работающих в условиях установившейся ползучести при поперечном и продольно-поперечном нагружениях. В инженерной практике помимо пластин в качестве тонкостенных элементов конструкций широко используются оболочки. Поэтому актуальной является проблема расчёта металлокомпозитных оболочечных элементов при установившейся ползучести всех фаз композиции. Изучению этого вопроса применительно к слоистым пологим оболочкам со сложными структурами армирования посвящено настоящее исследование.

1. Исходные уравнения и граничные условия. Рассмотрим деформирование тонкой пологой оболочки, подчиняющейся гипотезам Кирхгофа-Лява и состоящей из армированных и изотропных слоев переменной толщины. Допустимость гипотез Кирхгофа-Лява при расчёте установившейся ползучести тонкостенных элементов конструкций обоснована в [3]. Предполагается, что к рассматриваемому моменту времени деформации ползучести получили настолько значительное развитие, что по сравнению с ними можно пренебречь начальными упругими и пластическими деформациями [3]; по толщине каждый слой имеет регулярную и квазиоднородную структуру; на границах между слоями выполняются условия идеального механического контакта; перемещения считаются малыми, т. е. принимается геометрически линейная теория оболочек; температура $T$ конструкции однородна по её толщине и известна из решения соответствующей стационарной задачи теплопроводности [4].

С пологой оболочкой связана ортогональная система координат $x_{1} x_{2} z$;

Андрей Петрович Янковский (д.ф.-м.н.), ведущий научный сотрудник, лаб. физики быстропротекающих процессов. 
поверхность $x_{1} x_{2}(z=0)$ - отсчётная, причем координатные линии $x_{1}, x_{2}$ совпадают с линиями главной кривизны этой поверхности, а ось $z$ перпендикулярна отсчётной поверхности. Конструкция состоит из $M$ слоев, которые пронумеруем последовательно, начиная от нижней лицевой поверхности. Лицевые поверхности оболочки и границы между слоями задаются равенствами $z=Z_{m}\left(x_{1}, x_{2}\right)(0 \leqslant m \leqslant M)$, причём функции $Z_{0}, Z_{M}$ определяют нижнюю и верхнюю лицевые поверхности соответственно, а $Z_{m}$ - поверхность контакта $m$-го и $(m+1)$-того слоев $(1 \leqslant m \leqslant M-1)$. Выполняются неравенства $Z_{0} \leqslant Z_{1} \leqslant Z_{2} \leqslant \ldots \leqslant Z_{m} \leqslant \ldots \leqslant Z_{M}$

Каждый $m$-тый слой армирован $K^{(m)}$ семействами волокон (металлических проволок, возможно, различной физической природы), которые уложены по поверхностям, эквидистантным отсчётной поверхности (эквидистантное армирование), или по поверхностям, расстояния между которыми по оси $z$ изменяются пропорционально изменению толщины слоя $Z_{m}\left(x_{1}, x_{2}\right)-$ - $Z_{m-1}\left(x_{1}, x_{2}\right)$ (неэквидистантное армирование).

Для формулировки задачи деформирования в условиях установившейся ползучести металлокомпозитных пологих оболочек слоисто-волокнистой структуры используем общеизвестные [3, 5]

- уравнения равновесия:

$$
\begin{aligned}
& \left(A_{j} M_{i i}\right)_{, i}-A_{j, i} M_{j j}+\left(A_{i} M_{j i}\right)_{, j}+A_{i, j} M_{i j}-A_{1} A_{2} Q_{i}+A_{1} A_{2} m_{i}=0, \\
& \left(A_{j} F_{i i}\right)_{, i}-A_{j, i} F_{j j}+\left(A_{i} F_{j i}\right)_{, j}+A_{i, j} F_{i j}+A_{1} A_{2} q_{i}=0 \quad(j=3-i, i \equiv 1,2) ; \\
& \left(A_{2} Q_{1}\right)_{, 1}+\left(A_{1} Q_{2}\right)_{, 2}-A_{1} A_{2}\left(F_{11} / R_{1}+F_{22} / R_{2}\right)+A_{1} A_{2} q_{z}=0 ;
\end{aligned}
$$

- соотношения, связывающие скорости мембранных деформаций $\dot{e}_{i j}$ и параметров искривления $\dot{\kappa}_{i j}$ отсчётной поверхности оболочки со скоростями перемещений $\dot{u}_{i}, \dot{w}$ этой поверхности:

$$
\begin{aligned}
& \dot{e}_{i i}=A_{i}^{-1} \dot{u}_{i, i}+\left(A_{1} A_{2}\right)^{-1} A_{i, j} \dot{u}_{j}+R_{i}^{-1} \dot{w} \\
& 2 \dot{e}_{12}=2 \dot{e}_{21}=A_{1} A_{2}^{-1}\left(\dot{u}_{1} / A_{1}\right)_{, 2}+A_{2} A_{1}^{-1}\left(\dot{u}_{2} / A_{2}\right)_{, 1}, \\
& \dot{\kappa}_{i i}=-A_{i}^{-1}\left(\dot{w}_{, i} / A_{i}\right)_{, i}-A_{j}^{-2} A_{i}^{-1} A_{i, j} \dot{w}_{, j} \quad(j=3-i, i \equiv 1,2) ; \\
& \dot{\kappa}_{12}=\dot{\kappa}_{21}=-\left(A_{1} A_{2}\right)^{-1}\left(\dot{w}_{, 12}-A_{1,2} \dot{w}_{, 1} / A_{1}-A_{2,1} \dot{w}_{, 2} / A_{2}\right) ;
\end{aligned}
$$

- определяющие соотношения, связывающие мембранные усилия $F_{i j}$ и моменты $M_{i j}$ в оболочке со скоростями деформаций $\dot{e}_{i j}$ и параметров $\dot{\kappa}_{i j}(i \equiv 1,2$, $j \equiv 1,2)$ :

$$
\begin{aligned}
& F_{i j}=\sum_{n=1}^{2} \sum_{l=1}^{2}\left(A_{i j n l} \dot{e}_{n l}+B_{i j n l} \dot{\kappa}_{n l}\right), \\
& M_{i j}=\sum_{n=1}^{2} \sum_{l=1}^{2}\left(D_{i j n l} \dot{e}_{n l}+C_{i j n l} \dot{\kappa}_{n l}\right),
\end{aligned}
$$

где коэффициенты $A_{i j n l}, B_{i j n l}, C_{i j n l}, D_{i j n l}$ в рамках теории течения установившейся ползучести [3], используемой в настоящем исследовании, нелинейно зависят от $\dot{e}_{n l}$ и $\dot{\kappa}_{n l}$, параметров армирования, температуры $T\left(x_{1}, x_{2}\right)$, толщины слоев и механических характеристик установившейся ползучести фазовых материалов.

Так как тонкая пологая оболочка подчиняется гипотезам Кирхгофа-Лява, то для определения $A_{i j n l}, B_{i j n l}, C_{i j n l}, D_{i j n l}$ можно использовать соотношения, полученные в [2] (не будем их здесь приводить в силу их громоздкости). 
В случае неэквидистантного армирования тонкого $m$-того слоя непрерывными волокнами постоянного поперечного сечения между параметрами армирования существует связь, приближенно определяемая равенством [6]

$$
\begin{aligned}
\left(A _ { 2 } \omega _ { k } ^ { ( m ) } \left(Z_{m}-\right.\right. & \left.\left.Z_{m-1}\right) \cos \psi_{k}^{(m)}\right)_{, 1}+ \\
& +\left(A_{1} \omega_{k}^{(m)}\left(Z_{m}-Z_{m-1}\right) \sin \psi_{k}^{(m)}\right)_{, 2}=0, \quad 1 \leqslant k \leqslant K^{(m)}
\end{aligned}
$$

которое при эквидистантном армировании редуцируется в точное равенство, получающееся из (4) формальным заданием $Z_{m}-Z_{m-1}=1$.

$\mathrm{K}$ уравнениям и соотношениям (1)-(3) необходимо добавить общеизвестные граничные условия на кромках оболочки [5] (не будем их здесь приводить, так как они весьма разнообразны).

На той части кромки (обозначим ее $\Gamma_{k}^{(m)}$ ), на которой волокна $k$-того семейства $m$-того слоя, имеющие постоянные поперечные сечения, входят в конструкцию, необходимо задать краевые условия [6]:

$$
\omega_{k}^{(m)}\left(\Gamma_{k}^{(m)}\right)=\omega_{0 k}^{(m)}, \quad 1 \leqslant k \leqslant K^{(m)}, \quad 1 \leqslant m \leqslant M .
$$

В уравнениях и соотношениях (1)-(5) приняты следующие обозначения: $A_{i}, R_{i}$ - параметры Ламе и главные радиусы кривизны отсчётной поверхности оболочки соответственно; $Q_{i}$ - перерезывающие усилия; $q_{i}, q_{z}$ - приведенные распределённые нагрузки в направлениях $x_{i}, z$ соответственно; $m_{i}$ приведённые распределённые изгибающие моменты, вызванные внешними нагрузками; $\dot{w}, \dot{u}_{i}-$ скорость прогиба и скорости перемещений точек отсчётной поверхности оболочки соответственно в направлениях $x_{i}(i \equiv 1,2)$ в условиях установившейся ползучести; $\omega_{k}^{(m)}, \psi_{k}^{(m)}$ - интенсивность и угол (отсчитываемый от направления координатной линии $x_{1}$ ) армирования $m$-того слоя волокнами $k$-того семейства; $\omega_{0 k}^{(m)}$ - заданная на кромке $\Gamma_{k}^{(m)}$ функция; нижний индекс после запятой означает частное дифференцирование по соответствующей переменной $x_{i}$; точка над функцией означает частную производную от этой функции по времени.

2. Система разрешающих уравнений. Для получения системы разрешающих уравнений деформирования слоистых армированных пологих оболочек, работающих в условиях установившейся ползучести, и соответствующих ей (системе) статических граничных условий в скоростях прогиба $\dot{w}$ и тангенциальных перемещений $\dot{u}_{i}$ необходимо выражения для $\dot{e}_{i j}, \dot{\kappa}_{i j}$ из (2) подставить в соотношения (3), а последние - в уравнения равновесия (1) и граничные условия и исключить из рассмотрения поперечные силы $Q_{i}$. Тогда три уравнения равновесия примут разрешающий вид

$$
\begin{gathered}
\left(A_{j} \Lambda_{i i}^{(1)}\right)_{, i}-A_{j, i} \Lambda_{j j}^{(1)}+\left(A_{j} \Lambda_{i j}^{(1)}\right)_{, j}+A_{i, j} \Lambda_{i j}^{(1)}=-A_{1} A_{2} q_{i} \\
j=3-i, i \equiv 1,2 \\
\sum_{i=1}^{2}\left\{A_{i}^{-1}\left[A_{1} A_{2} m_{i}+\left(A_{j} \Lambda_{i i}^{(2)}\right)_{, i}-A_{j, i} \Lambda_{j j}^{(2)}+\left(A_{i} \Lambda_{i j}^{(2)}\right)_{, j}+A_{i, j} \Lambda_{i j}^{(2)}\right]\right\}, i- \\
-A_{1} A_{2} \sum_{i=1}^{2} R_{i}^{-1} \Lambda_{i i}^{(1)}=-A_{1} A_{2} q_{z}, \quad j=3-i,
\end{gathered}
$$


где

$$
\begin{gathered}
\Lambda_{i j}^{(1)}=\sum_{l=1}^{2}\left(A_{i j l l}\left(A_{l}^{-1} \dot{u}_{l, l}+\left(A_{1} A_{2}\right)^{-1} A_{l, m} \dot{u}_{m}+R_{l}^{-1} \dot{w}\right)-\right. \\
\left.-B_{i j l l}\left(A_{l}^{-1}\left(A_{l}^{-1} \dot{w}_{, l}\right)_{, l}+A_{m}^{-2} A_{l}^{-1} A_{l, m} \dot{w}_{, m}\right)\right)+ \\
+A_{i j 12}\left(A_{1} A_{2}^{-1}\left(A_{1}^{-1} \dot{u}_{1}\right)_{, 2}+A_{2} A_{1}^{-1}\left(A_{2}^{-1} \dot{u}_{2}\right)_{, 1}\right)- \\
-2 B_{i j 12}\left(A_{1} A_{2}\right)^{-1}\left(\dot{w}_{, 12}-A_{1}^{-1} A_{1,2} \dot{w}_{, 1}-A_{2}^{-1} A_{2,1} \dot{w}_{, 2}\right), \quad m=3-l ; \\
\Lambda_{i j}^{(2)}=\sum_{l=1}^{2}\left(D_{i j l l}\left(A_{l}^{-1} \dot{u}_{l, l}+\left(A_{1} A_{2}\right)^{-1} A_{l, m} \dot{u}_{m}+R_{l}^{-1} \dot{w}\right)-\right. \\
\left.-C_{i j l l}\left(A_{l}^{-1}\left(A_{l}^{-1} \dot{w}_{, l}\right)_{, l}+A_{m}^{-2} A_{l}^{-1} A_{l, m} \dot{w}_{, m}\right)\right)+ \\
+D_{i j 12}\left(A_{1} A_{2}^{-1}\left(A_{1}^{-1} \dot{u}_{1}\right)_{, 2}+A_{2} A_{1}^{-1}\left(A_{2}^{-1} \dot{u}_{2}\right)_{, 1}\right)- \\
-2 C_{i j 12}\left(A_{1} A_{2}\right)^{-1}\left(\dot{w}_{, 12}-A_{1}^{-1} A_{1,2} \dot{w}_{, 1}-A_{2}^{-1} A_{2,1} \dot{w}_{, 2}\right), \quad m=3-l .
\end{gathered}
$$

В теории установившейся ползучести тонких слоистых оболочек статические граничные условия на кромке $x_{i}=$ const могут быть заданы с помощью линейных комбинаций следующих величин $[3,5]$ :

$$
\begin{array}{r}
\Lambda_{i i}^{(1)}=F_{i i}^{0}, \quad \Lambda_{i i}^{(2)}=M_{i i}^{0}, \quad \Lambda_{i j}^{(1)}+R_{j}^{-1} \Lambda_{i j}^{(2)}=F_{i j}^{0}, \\
A_{1} A_{2} m_{i}+\left(A_{j} \Lambda_{i i}^{(2)}\right)_{, i}-A_{j, i} \Lambda_{j j}^{(2)}+\left(A_{i} \Lambda_{i j}^{(2)}\right)_{, j}+A_{i, j} \Lambda_{i j}^{(2)}+ \\
+A_{i} \Lambda_{i j, j}^{(2)}=A_{1} A_{2} Q_{i}^{0}, \quad j=3-i, i \equiv 1,2,
\end{array}
$$

где $F_{i i}^{0}, M_{i i}^{0}, F_{i j}^{0}, Q_{i}^{0}$ - заданные на кромке $x_{i}=$ const силовые факторы $\left(Q_{i}^{0}-\right.$ приведённая сила Кирхгофа).

Кинематические же граничные условия на кромке $x_{i}=$ const могут быть заданы с помощью линейных комбинаций величин $[3,5]$

$$
\dot{u}_{l}=\dot{u}_{l}^{0}, \quad \dot{w}=\dot{w}_{0}, \quad-A_{i}^{-1} \dot{w}_{, i}+R_{i}^{-1} \dot{u}_{i}=\dot{\vartheta}_{i}^{0}, \quad l \equiv 1,2,
$$

где $\dot{u}_{l}^{0}, \dot{w}_{0}, \dot{\vartheta}_{i}^{0}$ - заданные на кромке функции (скорости перемещений и углов поворотов).

Кроме того, граничные условия могут быть заданы также в смешанном из (8), (9) виде.

В случае, когда контур, ограничивающий отсчётную поверхность пологой оболочки, не совпадает с одной из координатных линий $x_{i}=$ const, статические и кинематические граничные условия, записанные в скоростях перемещений, существенно усложняются [5] по сравнению с (8), (9), поэтому не будем этот случай рассматривать более подробно (в рамках настоящего исследования это не принципиально).

Если аппликаты границ $Z_{m}$ и траектории армирования (т. е. углы $\psi_{k}^{(m)}$ ) слоёв заданы, то краевая задача $(4),(5)$ определяет интенсивность $\omega_{k}^{(m)}$ aрмирования $m$-того слоя волокнами $k$-того семейства. Краевые задачи для линейных уравнений в частных производных первого порядка (4) хорошо изучены [7], поэтому не будем останавливаться на обсуждении этого вопроса более 
подробно; отметим лишь, что уравнение (4) имеет действительные характеристики, совпадающие с траекториями армирования $k$-того семейства $m$-того слоя. Если функции $\psi_{k}^{(m)}, \omega_{k}^{(m)}, Z_{m}$ известны (заданы), то в оболочке можно определить стационарное температурное поле $T\left(x_{1}, x_{2}\right)$ (соответствующие граничные задачи сформулированы и проанализированы, например, в [4]), после чего три разрешающих уравнения равновесия (6), (7) будут замкнуты относительно трёх функций $\dot{u}_{1}, \dot{u}_{2}, \dot{w}$. Этой системе квазилинейных дифференциальных уравнений эллиптического типа соответствуют нелинейные (8) и линейные (9) граничные условия (или их комбинации).

Основная трудность, возникающая при решении сформулированной граничной задачи деформирования слоисто-волокнистых пологих оболочек в условиях установившейся ползучести, заключается в её существенной нелинейности. Для линеаризации этой задачи можно использовать итерационный процесс, основанный на применении метода секущего модуля [3], адаптация которого к расчёту тонкостенных металлокомпозитных элементов конструкций подробно изложена в [2]. Сходимость метода секущего модуля доказана в [3]. Так как на каждой итерации такого процесса приходится решать линейную граничную задачу, формально схожую [3] с задачей упругого деформирования слоистых анизотропных и неоднородных пологих оболочек со слоями переменной толщины $[5,8]$, то для интегрирования такой граничной задачи можно использовать уже известные и хорошо разработанные конечно-разностные схемы, методы конечных элементов или другие приближенные вариационные методы. Не будем останавливаться на обсуждении этого вопроса более подробно.

З А м Е ч А н и Е. Зная из сформулированной граничной задачи скорости прогиба $\dot{w}$ и тангенциальных перемещений точек отсчётной поверхности $\dot{u}_{1}$, $\dot{u}_{2}$, по формулам (2), (3) можно определить внутренние силовые факторы $F_{i j}$, $M_{i j}$ в оболочке (а по формулам $(1.2),(1.6)-(1.9),(1.17)-(1.19)$ из [2] - напряженное состояние во всех фазовых материалах и в композиции каждого слоя в целом), работающей в условиях установившейся ползучести. Если известно помимо этого и начальное состояние для внутренних силовых факторов $\bar{F}_{i j}$, $\bar{M}_{i j}$ (порождаемое осреднёнными напряжениями $\bar{\sigma}_{i j}^{(m)}$ в каждом $m$-том слое, см. (1.2), (1.3) в [9]) в конструкции (его можно определить методами, изложенными, например, в $[5,8])$, то решение о неустановившейся ползучести на первой её стадии можно получить приближенно, используя методику, предложенную в [3]. Для этого представим текущее состояние для внутренних силовых факторов в оболочке в виде

$$
\overline{\bar{F}}_{i j}=(1-\tau(t)) \bar{F}_{i j}+\tau(t) F_{i j}, \quad \overline{\bar{M}}_{i j}(1-\tau(t)) \bar{M}_{i j}+\tau(t) M_{i j},
$$

а соответствующее ему текущее осреднённое напряженное состояние в $m$-том слое -

$$
\overline{\bar{\sigma}}_{i j}^{(m)}=(1-\tau(t)) \bar{\sigma}_{i j}^{(m)}+\tau(t) \sigma_{i j}^{(m)}, \quad i \equiv 1,2, \quad j \equiv 1,2, \quad 1 \leqslant m \leqslant M,
$$

где $\tau(t)$ - монотонная функция времени $t$, возрастающая от значения $\tau=0$ в начальный момент времени $t=0$ до $\tau=1$ при $t \rightarrow \infty$. Функцию $\tau(t)$ можно определить исходя из экстремальных принципов теории ползучести (см. § 34 в [3]). В случае изотропных оболочек реализация такого подхода не вызывает 
особых трудностей, а в случае слоистых конструкций со сложными структурами армирования требует проведения дополнительного, более тщательного исследования, выходящего за рамки настоящей работы.

3. Модельная задача. В качестве модельной задачи рассмотрим осесимметричное деформирование сферической (радиуса $R$ ) пологой оболочки в условиях установившейся ползучести, занимающей в плане кольцевую область, ограниченную контурами радиусов $r_{0}, r_{1}\left(r_{0}<r_{1}\right)$. Конструкция осесимметрично нагружена (нагрузки в окружном направлении $x_{2}$ отсутствуют) и нагрета $\left(T=T\left(x_{1}\right)\right)$. Армирование каждого $m$-того слоя осуществляется чётным количеством семейств волокон $\left(K^{(m)}=2 N^{(m)}\right)$ по меридиональносимметричным траекториям $\left(\psi_{2 k-1}^{(m)}\left(x_{1}\right)=-\psi_{2 k}^{(m)}\left(x_{1}\right), \omega_{2 k-1}^{(m)}\left(x_{1}\right)=\omega_{2 k}^{(m)}\left(x_{1}\right)\right.$, $\left.1 \leqslant k \leqslant N^{(m)}, 1 \leqslant m \leqslant M\right)$.

В силу осесимметричного термосилового нагружения такой оболочки, отсутствия нагрузок в окружном направлении и меридионально-симметричного её армирования тождественно равны нулю функции $\dot{u}_{2}, \dot{e}_{12}, \dot{\kappa}_{12}, M_{12}$, $F_{12}$, поэтому уравнения равновесия (1) редуцируются в следующую систему $\left(x_{1} \equiv r\right)$ :

$$
\begin{aligned}
& F_{11}^{\prime}+\left(F_{11}-F_{22}\right) / r=-q_{1}(r, \boldsymbol{\omega}, \boldsymbol{Z}), \\
& M_{11}^{\prime}+\left(M_{11}-M_{22}\right) / r=Q_{1}, \\
& Q_{1}^{\prime}+Q_{1} / r-\left(F_{11}+F_{22}\right) / R=-q_{z}(r, \boldsymbol{\omega}, \boldsymbol{Z}), \\
& \boldsymbol{\omega}=\left\{\omega_{1}^{(1)}, \omega_{2}^{(2)}, \ldots, \omega_{K^{(M)}}^{(M)}\right\}, \quad \boldsymbol{Z}=\left\{Z_{0}, Z_{1}, \ldots, Z_{M}\right\},
\end{aligned}
$$

где остальные уравнения выполняются тождественно; штрих означает производную по полярному радиусу $r$.

K уравнениям (10) нужно добавить геометрические соотношения (см. (2))

$$
\dot{e}_{11}=\dot{u}_{1}^{\prime}+\dot{w} / R, \quad \dot{e}_{22}=\dot{u}_{1} / r+\dot{w} / R, \quad \dot{\kappa}_{11}=-\dot{w}^{\prime \prime}, \quad \dot{\kappa}_{22}=-\dot{w}^{\prime} / r
$$

и определяющие уравнения (см. (3))

$$
F_{i i}=\sum_{j=1}^{2}\left(A_{i i j j} \dot{e}_{j j}+B_{i i j j} \dot{\kappa}_{j j}\right), \quad M_{i i}=\sum_{j=1}^{2}\left(D_{i i j j} \dot{e}_{j j}+C_{i i j j} \dot{\kappa}_{j j}\right), \quad i \equiv 1,2 .
$$

При жёстком защемлении кромок оболочки

$$
\dot{u}_{1}(r)=0, \quad \dot{w}(r)=0, \quad \dot{w}^{\prime}(r)=0 \quad\left(r=r_{0}, r=r_{1}\right),
$$

в случае же свободной от нагружения кромки

$$
F_{11}(r)=0, \quad M_{11}(r)=0, \quad Q_{1}(r)=0 \quad\left(r=r_{0}, r=r_{1}\right) .
$$

Отметим, что могут быть заданы и другие граничные условия, например шарнирное опирание кромок и т. п.

Используя процедуру, изложенную в [5], уравнения (10)-(12) можно свести к системе обыкновенных дифференциальных уравнений первого порядка, разрешенную относительно производных по $r$. Линеаризовав эту систему указанным выше методом, соответствующую двухточечную граничную задачу 
(например, при граничных условиях (13), (14)) можно численно проинтегрировать методом пристрелки с применением дискретной ортогонализации [5].

3. Обсуждение результатов расчётов. В качестве примера исследуем установившуюся ползучесть однослойных и трёхслойных сферических пологих оболочек постоянной толщины $2 H=Z_{M}-Z_{0}=2 \mathrm{~cm}$, кромки которых в плане определяются радиусами $r_{0}=0,1$ м и $r_{1}=0,5$ м. Внутренние кромки $\left(r=r_{0}\right)$ оболочек жёстко защемлены (см. (13)), а внешние кромки $\left(r=r_{1}\right)$ свободны от нагружения (см. (14)). На оболочки действует равномерная поперечная нагрузка (давление) $q_{z}=-0,3 \mathrm{MПа}\left(q_{1} \equiv 0\right)$.

Радиус $R$ сферической оболочки связан со стрелой подъема $f$ полюсной точки отсчётной поверхности над плоскостью, определяющей нижнюю (внешнюю) кромку оболочки $\left(r=r_{1}\right)$, следующей формулой

$$
R=\frac{1}{2 f}\left(r_{1}^{2}+f^{2}\right) .
$$

Рассматриваются пологие металлокомпозитные оболочки двух типов: 1) однослойные оболочки $(M=1)$ с регулярной и квазиоднородной по толщине структурой, изготовленные из жёсткой меди $(\mathrm{Cu})$ или алюминиевого сплава АМг3 и меридионально-симметрично армированные по логарифмическим спиралям $\left(\omega_{1}^{(1)}(r)=\omega_{2}^{(1)}(r), \psi_{1}^{(1)}(r)=-\psi_{2}^{(1)}(r)=\psi=\right.$ const) двумя $\left(K^{(1)}=2\right)$ семействами стальной проволоки У8А; 2$)$ трёхслойные оболочки $(M=3)$, внутренний слой которых является изотропным и изготовлен из меди или сплава АМг3, а внешние слои постоянной толщины изготовлены из тех же материалов и меридионально-симметрично армированы по логарифмическим спиралям $\left(\omega_{1}^{(m)}(r)=\omega_{2}^{(m)}(r), \psi_{1}^{(m)}(r)=-\psi_{2}^{(m)}(r)=\psi=\right.$ const $)$ двумя $\left(K^{(m)}=2, m \equiv 1,3\right)$ семействами проволоки У8А.

При температуре $T \approx 200^{\circ} \mathrm{C}$ законы установившейся ползучести для этих материалов подчиняются следующим соотношениям:

$\mathrm{Cu}[10]: \quad \dot{\varepsilon}=B \sigma^{m}[1 / \mathrm{\Psi}], \quad B=3,65 \cdot 10^{-10}(\mathrm{M \Pi а})^{-m} \cdot \mathrm{\Psi}^{-1}, m=1,6 ;$ АМг3 [11] : $\dot{\varepsilon}=\exp (-K+\beta \sigma)[1 / \mathrm{\Psi}], K=12,2, \beta=0,0673(\mathrm{M \Pi а})^{-1}$;

Y8A [12] : $\dot{\varepsilon}=B \sigma^{m}[1 / \mathrm{\Psi}], \quad B=1,054 \cdot 10^{-84}(\mathrm{M \Pi a})^{-m} \cdot \mathrm{ч}^{-1}, m=24,982$.

Согласно [2], аппроксимируем эти зависимости двухзвенными кусочнолинейными функциями

$$
\sigma(\dot{\varepsilon})=\left\{\begin{array}{cc}
B_{1} \dot{\varepsilon}, & 0 \leqslant \dot{\varepsilon} \leqslant \dot{\varepsilon}_{1}, \\
\sigma_{1}+B_{2}\left(\dot{\varepsilon}-\dot{\varepsilon}_{1}\right), & \dot{\varepsilon}>\dot{\varepsilon}_{1}
\end{array}\right.
$$

с характеристиками для выбранных фазовых материалов

$$
\begin{aligned}
& \mathrm{Cu}: \quad B_{1}=4,754 \cdot 10^{13} \text { Па } \cdot \text { ч, } B_{2}=1,797 \cdot 10^{13} \text { Па } \cdot \text { ч, } \\
& \sigma_{1}=1,136 \text { ГПа, } \dot{\varepsilon}_{1}=2,39 \cdot 10^{-5} 1 / \text { ч; } \\
& \text { АМг3 : } \quad B_{1}=940,97 \text { ГПа •ч, } B_{2}=209,73 \text { ГПа } \\
& \sigma_{1}=30,194 \mathrm{M \Pi а}, \dot{\varepsilon}_{1}=3,209 \cdot 10^{-5} 1 / \text { ч; } \\
& \text { У8A : } \quad B_{1}=1,611 \cdot 10^{15} \text { Па } \cdot \text { ч, } B_{2}=1,257 \cdot 10^{12} \text { Па } \cdot \text { ч, } \\
& \sigma_{1}=1,466 \text { ГПа, } \dot{\varepsilon}_{1}=9,097 \cdot 10^{-7} 1 / \text { ч. }
\end{aligned}
$$




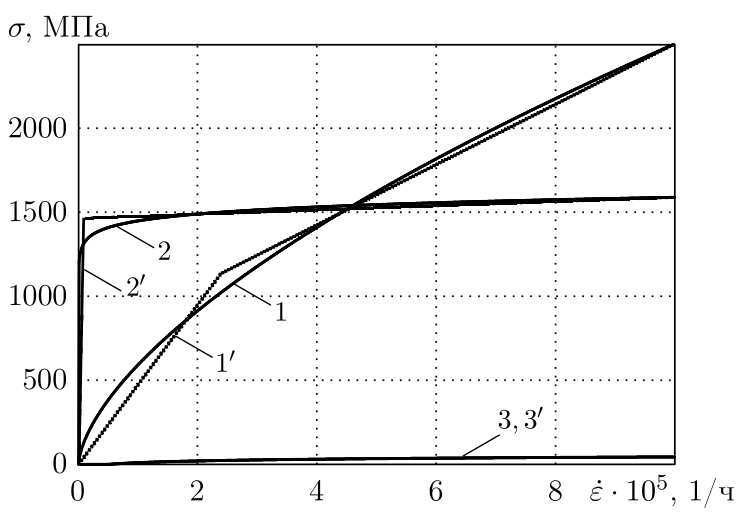

Рис. 1. Кривые установившейся ползучести фазовых материалов и их аппроксимация двухзвенными ломаными
Параметры (18) определены на основании зависимостей (16) методом наименьших квадратов на интервале $0 \leqslant \dot{\varepsilon} \leqslant 10^{-4} 1 /$ ч, при этом варьировались величины $\sigma_{1}, \dot{\varepsilon}_{1}$. Значения (18) можно несколько уточнить, если в (17) варьировать параметры $\sigma_{1}, \dot{\varepsilon}_{1}$, $B_{2}$. На рис. 1 сплошные кривые $1,2,3$ характеризуют зависимости (16) для материалов $\mathrm{Cu}, \mathrm{У8 \textrm {A } , ~ А М г 3 ~ с о о т в е т с т в е н н о ; ~}$ двухзвенные же штриховые ломаные $1^{\prime}, 2^{\prime}, 3^{\prime}$ характеризуют аппроксимации (17), (18) соответственно для тех же материа-

лов (в силу выбранного на рис. 1 масштаба по оси ординат линия $3^{\prime}$ визуально практически не отличается от 3 ). Сравнение кривых $1,2,3$ с линиями $1^{\prime}, 2^{\prime}, 3^{\prime}$ соответственно показывает, что зависимость (17) с параметрами (18) удовлетворительно аппроксимирует законы установившейся ползучести (16), поэтому при решении рассматриваемой задачи для вычисления коэффициентов $A_{i j n l}, B_{i j n l}, C_{i j n l}, D_{i j n l}$ в определяющих уравнениях (3), (12) можно обоснованно использовать методику, разработанную в [2].

В качестве критерия сопоставимости пологих оболочек с разными структурами армирования используем условие равенства в них общего расхода арматуры

$$
\Omega=\sum_{m=1}^{M}\left(Z_{m}-Z_{m-1}\right) \sum_{k=1}^{K^{(m)}} \int_{r_{0}}^{r_{1}} \omega_{k}^{(m)}(r) r d r \quad\left(K^{(m)}=2, M=1 \text { или } M=3\right) .
$$

Так как предполагается, что армированные слои имеют постоянную толщину, а проволока не обрывается внутри оболочек и имеет постоянное поперечное сечение, плотность армирования для рассматриваемых структур изменяется по закону [6] (случай эквидистантного армирования)

$$
\begin{array}{cl}
\omega_{k}^{(m)}(r)=r_{0} \omega_{0 k}^{(m)} / r, & \omega_{0 k}^{(m)}=\omega_{k}^{(m)}\left(r_{0}\right)=\mathrm{const}, \quad r_{0} \leqslant r \leqslant r_{1}, k \equiv 1,2, \\
\omega_{01}^{(m)}=\omega_{02}^{(m)}, & m=1 \text { при } M=1, \quad m \equiv 1,3 \text { при } M=3 .
\end{array}
$$

Для однослойных оболочек в расчётах примем $\omega_{01}^{(1)}=\omega_{02}^{(1)}=0,2$, а для армированных слоев трёхслойных конструкций $\omega_{0 k}^{(1)}=\omega_{0 k}^{(3)}=0,35(k \equiv 1,2)$, т. е. $\omega_{01}^{(m)}+\omega_{02}^{(m)}=0,7(m \equiv 1,3)$, что на практике соответствует удельной суммарной плотности армирования, близкой к предельно допустимой. Чтобы общий расход арматуры (19) в трёхслойных пологих оболочках с учётом (20) был такой же, как в однослойных оболочках, толщину $2 h$ внутреннего (неармированного) слоя в трёхслойных конструкциях нужно задать так:

$$
2 h=4(0,35-0,2) H / 0,7=6 H / 7 \quad(2 H=2 \mathrm{~cm}) .
$$


На рис. 2 изображены зависимости $\dot{w}_{*}(\psi), \dot{u}_{*}(\psi)$, где по оси ординат отложены скорости прогибов $\dot{w}$ и меридиональных смещений $\dot{u}_{1}$, определённые в точках внешней кромки $\left(\dot{w}_{*} \equiv\right.$ $\left.\dot{w}\left(r_{1}\right), \dot{u}_{*} \equiv \dot{u}_{1}\left(r_{1}\right)\right)$, что соответствует максимальным по модулю скоростям прогибов и меридиональных смещений точек отсчётных поверхностей тонкостенных конструкций при фиксированных структурам армирования (заданных $\psi$ ). На рис. 2, а приведены результаты расчётов пластин и пологих оболочек из $\mathrm{Cu}-\mathrm{У} 8 \mathrm{~A}-$-композиции, а на рис. 2, б-из АМг3-У8А-композиции. Кривые с одинаковыми номерами на рис. 2 характеризуют одни и те же зависимости, определённые при одних и тех же условиях, но для разных металлокомпозиций. Сплошные линии $1,2,1^{\prime \prime}, 2^{\prime \prime}$ на рис. 2 рассчитаны для пологих оболочек со стрелой подъема $f=6 \mathrm{~cm}$, а штриховые кривые $1^{\prime}, 2^{\prime}$ приведены для сравнения и получены для кольцевых пластин $(f=$ $=0)$. Линии $1^{\prime \prime}, 2^{\prime \prime}$ характеризу-

ют зависимости $\dot{u}_{*}(\psi)$, остальные кривые - зависимости $\dot{w}_{*}(\psi)$ (в пластинах в условиях заданного нагружения реализуется поперечный изгиб, поэтому в них $\dot{u}_{*}(\psi) \equiv 0$ и соответствующие линии не изображены). Кривые $1,1^{\prime}, 1^{\prime \prime}$ получены для однослойных тонкостенных конструкций, а линии $2,2^{\prime}, 2^{\prime \prime}$ для трёхслойных.

Кривые на рис. 2 характеризуют податливость рассматриваемых металлокомпозитных конструкций в условиях установившейся ползучести в зависимости от угла спирального армирования $\psi$ и разнесения несущих армированных слоёв. Поведение кривых на рис. 2 свидетельствует о существенной зависимости этой податливости от структуры армирования. Анализ поведения кривых на рис. 2 позволяет выбрать такие структуры армирования (из рассматриваемого класса), которые при заданном нагружении обеспечивают наибольшую или наименьшую податливость соответствующей конструкции в условиях установившейся ползучести; в частности, при рассматриваемых характерных размерах конструкций и уровне их нагружения наименьшие по модулю скорости прогиба и меридионального смещения при установившейся ползучести развиваются в меридионально (радиально) армированных $(\psi=0)$ 
оболочках и пластинах. С точки зрения минимизации податливости в условиях установившейся ползучести такие структуры армирования можно считать рациональными. Многочисленные расчёты, проведённые автором, показали, что при других условиях нагружения или при других характерных размерах пластин и пологих оболочек наименьшие по модулю скорости прогиба и тангенциальных смещений развиваются в конструкциях, армированных по другим направлениям $\psi \neq 0$ (об этом свидетельствует, например, правый участок кривой 1 на рис. 2, а). Следовательно, при разных видах и уровнях нагружения и при разных габаритах конструкций рациональными с точки зрения минимизации величин $\left|\dot{w}_{*}\right|,\left|\dot{u}_{*}\right|$ будут разные структуры армирования, которые в общем случае могут и не совпадать с рациональными структурами, определёнными на основе критериев кратковременной прочности [8].

На рис. 2 изображены зависимости $\dot{w}_{*}(\psi), \dot{u}_{*}(\psi)$ лишь для металлокомпозитных конструкций. Для изотропных же пластин и пологих оболочек той же геометрии, выполненных из меди, скорость прогиба имеет прядок $\left|\dot{w}_{*}\right| \approx 10^{2}$ мкм/ч, а для соответствующих конструкций из сплава АМг3$\left|\dot{w}_{*}\right| \approx 10^{3}$ мкм/ч. Сравнение этих значений со значениями ординат точек на кривых $1,1^{\prime}, 2,2^{\prime}$ рис. 2 позволяет заключить, что замена тонкостенных изотропных металлических конструкций из меди или сплава АМг3 на соответствующие армированные металлокомпозитные изделия приводит к резкому (в разы и на порядки) уменьшению их податливости в условиях установившейся ползучести.

Кривые $2,2^{\prime}$ на рис. 2 лежат всюду выше линий $1,1^{\prime}$ соответственно, а кривые $2^{\prime \prime}$, наоборот, - ниже линий $1^{\prime \prime}$. Следовательно, разнесение несущих армированных слоев при заданном типе нагружения конструкций позволяет существенно уменьшить податливость рассматриваемых пластин и пологих оболочек в условиях установившейся ползучести.

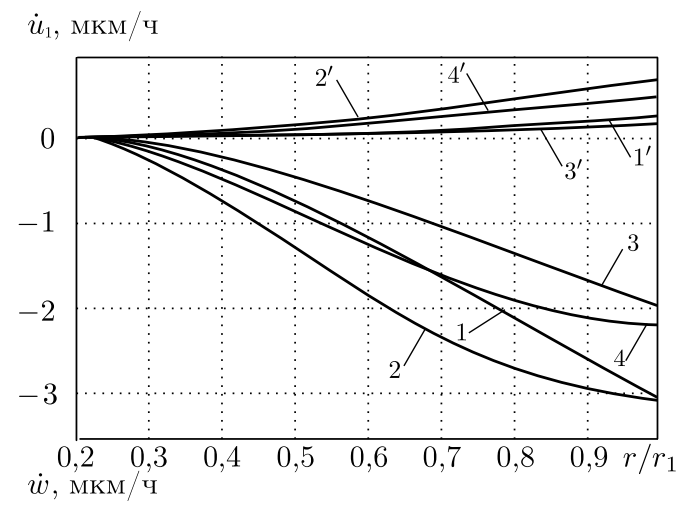

Рис. 3. Эпюры скоростей перемещений точек отсчётной поверхности пологой оболочки в условиях установившейся ползучести при четырёх структурах армирования

Ординаты точек на кривых рис. 2, а почти всюду по модулю меньше ординат точек на соответствующих кривых рис. 2,6 . Следовательно, в условиях установившейся ползучести податливость конструкций из $\mathrm{Cu}-\mathrm{У} 8 \mathrm{~A}$ композиции меньше податливости соответствующих изделий из АМг3-У8А-композиции, что вполне согласуется с поведением кривых установившейся ползучести 1,3 на рис. 1.

Изменение структуры армирования приводит не только к количественному, но и к некоторому качественному изменению поведения функций $\dot{w}(r), \dot{u}_{1}(r)$ в рассматриваемых конструкциях. Так, на рис. 3 приведены зависимости $\dot{w}$, $\dot{u}_{1}$ от безразмерного полярного радиуса $r / r_{1}$ для четырёх структур армирования пологой оболочки из $\mathrm{Cu}-\mathrm{У} 8 \mathrm{~A}$-композиции. Кривые $1,1^{\prime}, 2,2^{\prime}$ полу- 
чены для однослойной конструкции, а линии $3,3^{\prime}, 4,4^{\prime}$ - для трёхслойной; при этом кривые $1,1^{\prime}, 3,3^{\prime}$ определены при меридиональном армировании $(\psi=0)$, а линии $2,2^{\prime}, 4,4^{\prime}$ - при спиральном армировании $(\psi=2 \pi / 5)$.

Кривые 1, 2 на рис. 2 всюду лежат выше линий $1^{\prime}, 2^{\prime}$ соответственно, следовательно, искривление пластин (кривые $1^{\prime}$, $\left.2^{\prime}\right)$ приводит к уменьшению их податливости в условиях ползучести, по крайней мере, в нормальном к срединной поверхности направлении при поперечном нагружении. В связи с этим целесообразно проследить за изменением $\dot{w}_{*}, \dot{u}_{*}$ в зависимости от величины стрелы подъема $f$ пологой оболочки, которая связана с радиусом $R$ её кривизны равенством (15). На рис. 4 изображены зависимости $\dot{w}_{*}(f)$,

$\dot{u}_{*}, \mathrm{MKM} / \mathrm{\Psi}$

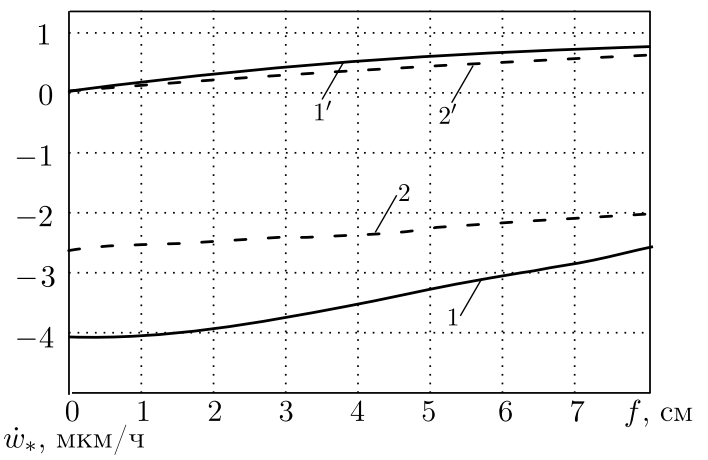

Рис. 4. Зависимости скоростей перемещений точек внешних кромок пологих оболочек в условиях установившейся ползучести от величины стрелы подъёма полюсной точки отсчётной поверхности $\dot{u}_{*}(f)$ для спирально $(\psi=2 \pi / 5)$ армированных однослойных (сплошные кривые $1,1^{\prime}$ ) и трёхслойных (штриховые линии $2,2^{\prime}$ ) пологих оболочек из $\mathrm{Cu}-У 8 \mathrm{~A}-$ композиции прежней толщины, прежней в плане геометрии и прежнего расхода арматуры. Кривые 1, 2 характеризуют зависимости $\dot{w}_{*}(f)$, а линии $1^{\prime}, 2^{\prime}-\dot{u}_{*}(f)$. Все кривые на рис. 4 монотонно возрастают. Поведение линии 2 показывает, что с увеличением стрелы подъема $f$ скорость прогиба установившейся ползучести трёхслойных пологих оболочек уменьшается по модулю незначительно (по крайней мере, при заданном уровне нагружения). Поведение же кривой 1, наоборот, свидетельствует о значительном уменьшении $\left|\dot{w}_{*}\right|$ однослойных оболочек рассматриваемой структуры при увеличении $f$. Следовательно, при определённых структурах армирования, видах и уровнях нагружения увеличение кривизны пологой оболочки (увеличение $f$ ) позволяет существенно снизить её податливость в условиях установившейся ползучести.

Заключение. Проведённый анализ деформирования металлокомпозитных пластин и пологих оболочек слоисто-волокнистой структуры, работающих в условиях установившейся ползучести, показывает, что армирование тонкостенной конструкции позволяет существенно уменьшить по модулю скорости перемещений точек отсчётной поверхности по сравнению с изотропными конструкциями той же геометрии, выполненных из материала связующей матрицы. При определённых видах нагружения таких конструкций разнесение несущих слоев позволяет дополнительно уменьшить податливость тонкостенных элементов изделий, работающих в условиях установившейся ползучести. При разных видах и уровнях нагружения тонкостенных конструкций и их размерах рациональными с точки зрения минимизации по модулю скоростей установившейся ползучести являются разные структуры армирования. Эффективные с точки зрения кратковременной прочности проекты армирования могут оказаться неэффективными с точки зрения поведения конструкции 
в условиях установившейся ползучести. При определённых структурах армирования увеличение кривизны пологой оболочки приводит к увеличению её сопротивляемости поперечному нагружению в условиях установившейся ползучести.

Работа выполнена при поддержке РФФИ (код проекта № 08-01-00046-а).

\section{БИБЛИОГРАФИЧЕСКИЙ СПИСОК}

1. Немировский Ю. В., Янковский А. П. Установившаяся ползучесть слоисто-волокнистых изгибаемых металлокомпозитных пластин // Вестн. Сам. гос. техн. ун-та. Сер. Физ.-мат. науки, 2008. - № 2(17). - С. 66-76.

2. Немировский Ю. В., Янковский А.П. Установившаяся ползучесть слоистых металлокомпозитных пластин со сложными структурами армирования при продольно-поперечном изгибе // Механика композиционных материалов и конструкиий, 2009. T. 45, № 1. - C. 59-82; англ. пер.: Nemirovskii Yu.V., Yankovskii A.P. The steady creeping compound reinforced metal-composites layer-plates at the transverse-longitudinal bending // Composite Mechanics and Design, 2009. - Vol. 45, No. 1. - P. 59-82.

3. Качанов Л. М. Теория ползучести. - М.: Физматгиз, 1960. - 456 с.

4. Немировский Ю. В., Янковский А. П. Теплопроводность волокнистых оболочек // Tenлофизика и аэромеханика, 1998. - Т. 5, № 2. - С. 215-235.

5. Григоренко Я. М. Изотропные и анизотропные слоистые оболочки вращения переменной жесткости. - Киев: Наукова думка, 1973. - 228 с.

6. Немировский Ю.В., Янковский А.П. О некоторых особенностях уравнений оболочек, армированных волокнами постоянного поперечного сечения // Механика композиционных материалов и конструкиий, 1997. - Т. 3, № 2. - С. 20-40; англ. пер.: Nemirovsky Yu. V., Yankovsky A.P. Certain properties of the equations of shells reinforced with constant cross section fibers // Composite Mechanics and Design, 1997. - Vol.3, No. 2. - P. 17-31.

7. Камке Э. Справочник по дифференциальным уравнениям в частных производных первого порядка. - М.: Наука, 1966. - 260 с.

8. Немировский Ю. В., Янковский А. П. Термоупругопластическое деформирование сложно армированных оболочек // Механика композитных материалов, 2006. - Т.42, №6. - C. 707-728; англ. пер.: Nemirovskii Yu. V., Yankovskii A.P. Thermoelastoplastic deformation of complexly reinforced shells // Mechanics of Composite Materials, 2006. Vol. 42, No. 6. - P. 491-506.

9. Немировский Ю. В., Янковский А. П. Термоупругопластический изгиб слоисто-волокнистых пластин// Механика композиционных материалов и конструкиий, 2005. T. 11, № 4. - C. 467-493.

10. Писаренко Г. С., Можаровский Н. С. Уравнения и краевые задачи теории пластичности и ползучести: Справ. пособ. - Киев: Наукова думка, 1981. - 496 с.

11. Соснин О. В. О ползучести слабо упрочняющихся материалов при нестационарных температурно-силовых режимах // Пробл. прочности, 1972. — № 1. - С. 74-77.

12. Композиционные материалы: Справочн. / ред. Д. М. Карпинос. - Киев: Наукова думка, 1985. - 592 с.

Поступила в редакцию 25/XI/2009;

в окончательном варианте - 15/III/2010. 
MSC: 74K20, 74R20

CALCULATION OF STEADY CREEPAGE OF METAL-COMPOSIT FLAT SHELLS OF LAYER-FIBROUS STRUCTURE

\section{A.P. Yankovskii}

Khristianovich Institute of Theoretical and Applied Mechanics,

Siberian Branch of the Russian Academy of Science,

4/1, Institutskaya str., Novosibirsk, 630090.

E-mails: nemirov@itam.nsc.ru, shulgin@itam.nsc.ru

The problem of deformation of flat layer-shells of the variable thickness reinforced by fibres of a constant cross-section and working in conditions of steady creepage of all phases of a composition is formulated. The system of the resolving equations and boundary conditions matching it is analysed. The problem-solving procedure in view is developed. The way of an approximate solution of such problems in conditions of unsteady creepage is specified. The specific calculations which have shown that compliance of thin-walled designs in conditions of the steady creepage essentially depends on structure of reinforcement were performed.

Key words: metal-composites, reinforcement, shells flat, plates, layer-fiber reinforcement, steady creepage.

Original article submitted $25 / \mathrm{XI} / 2009$; revision submitted 15/III/2010.

Andrey P. Yankovskii (Dr. Sci. (Phys. \& Math.)), Leading Research Scientist, Lab. of Fast Processes Physic. 